Notfall Rettungsmed 2015 · 18:527

DOI 10.1007/s10049-015-0044-6

Online publiziert: 20. August 2015

(c) Springer-Verlag Berlin Heidelberg 2015

CrossMark

J.D. Müller-Broich

Ltd. OA Sektion Wirbelsäulenchirurgie Klinik für Orthopädie, Dormagen, Deutschland

\title{
Transthorakale
}

\section{Echokardiographie und MRT der HWS allein ist nicht ausreichend}

sollte auch diesen Regionen eine besondere Aufmerksamkeit zukommen. Eitrige Infektionen an der Wirbelsäule sind, wie von den Autoren dargestellt, häufig übersehene Erkrankungen und werden oft erst in einem Spätstadium klinisch und radiologisch diagnostiziert. Zu diesem Zeitpunkt sind oft auch schon Gelenkersatz oder auch andere ossäre Implantate infiziert. Dies ist jedoch nicht regelhaft der Fall. Eine hohe Bedeutung kommt hier der 3-Phasen-Knochenszintigraphie zu, um auch implantatassoziierte Foci zu lokalisieren. Eine Punktion in Gelenke verbietet sich von selbst, besteht nicht der dringende klinische Verdacht einer Infektion in Verbindung mit einem bildmorphologischen Nachweis (Röntgen, Szintigraphie).

Das Fazit in Bezug auf die Praxis ist m. E. nicht ausreichend konsequent und aggressiv genug. Vielmehr sollte ein entsprechend qualifizierter Wirbelsäulenchirurg nicht erst bei Diagnosebestätigung, sondern bereits beim Verdacht hinzugezogen werden.

Schließlich bleibt anzumerken, dass nicht nur neurochirurgische Kollegen oder Fachabteilung zu einem solchen Fall hinzugezogen werden sollten. In Zeiten der interdisziplinären Entwicklungen im Bereich der Fachgesellschaften, in denen sich die Deutsche Wirbelsäulengesellschaft als Zusammenschluss von Neurochirurgen, Orthopäden und Unfallchirurgen mit Bezug zur operativen und aber natürlich auch konservativen Wirbelsäulenbehandlung versteht, kann der Hinweis zur Verlegung in eine Neurochirur- gie nicht zwingend als Fazit Ihrer Arbeit stehenbleiben.

Die Unterscheidung ist überholt.

Vielmehr sollte der Patient in eine Abteilung mit geeigneter operativer und auch konservativer Kompetenz im Bereich der Wirbelsäulenerkrankungen verlegt werden. $\mathrm{Ob}$ dies nun eine $\mathrm{NCH}$ (Neurochirurgie), Orthopädie oder Unfallchirurgie ist, sollte den lokalen Gegebenheiten überlassen werden. Hier gilt es, SOP zu entwickeln und konsequent umzusetzen.

Mit freundlichen Grüßen

Jacques D. Müller-Broich

\section{Korrespondenzadresse}

Dr. med. J.D. Müller-Broich

Ltd. OA Sektion Wirbelsäulenchirurgie Klinik für Orthopädie

Dr.-Geldmacher-Straße 20

45140 Dormagen

jdmbii@web.de
Zu diesem Leserbrief finden Sie auf der nächsten Seite eine Erwiderung. Diese finden Sie auch auf SpringerLink mit dem DOI 10.1007/s10049-015-0045-5 